\title{
Imaging of Fuel Cell and Battery Electrodes Using Focused Ion Beam Scanning Electron Microscopy
}

\author{
Scott Barnett ${ }^{1}$, Hongqian Wang, ${ }^{1}$ Zhao Liu, ${ }^{1}$ David Kennouche, ${ }^{1}$ and Kyle Yakal-Kremski ${ }^{1}$ \\ 1. Department Materials Science \& Engineering / Northwestern University, Evanston, IL, USA
}

This paper reviews results on the use of Focused Ion Beam - Scanning Electron Microscopy (FIB-SEM) for three-dimensional (3D) tomographic studies of fuel cell and battery electrode morphology. Electrodes are typically complex two- or three-phase (including porosity) structures where each phase transports a different species, and electrochemical reactions occur at the interfaces between phases. Thus, the electrode morphology plays an important role in determining the transport and reaction rates, and hence the electrochemical performance. 3D imaging has been used to understand electrochemical processes by determining macrohomogeneous parameters (surface areas, tortuosities, etc) and by utilizing measured 3D structures in 3D electrochemical simulations. Electrode electrochemical degradation has also been studied by relating observed morphological changes to degradation during accelerated testing - mechanistic models are developed to predict long-term performance behaviour.

Detailed studies of the 3D morphology of the most common SOFC electrodes, $\left(\mathrm{La}_{0.8} \mathrm{Sr}_{0.2}\right) \mathrm{MnO}_{3}$-Yttria Stabilized Zirconia (LSM-YSZ) cathodes [1-3] and Ni-YSZ anodes [4-8], have been carried out. In a recent study of LSM-YSZ, [3] cathodes fired at various temperatures, $T_{f}$, were studied using FIB-SEM and Electrochemical Impedance Spectroscopy (EIS). The total cathode polarization resistance, measured at $800^{\circ} \mathrm{C}$ in air by EIS, showed two dominant responses. The main EIS response, attributed to the oxygen reduction process, was minimized at $T_{f}=1175^{\circ} \mathrm{C}$. This correlated with an observed maximum in the density of electrochemically-active three-phase boundaries (TPBs) at this temperature, resulting from two factors: particle coarsening and densification at high $T_{f}$ that yielded a low TPB density, and low LSM percolation at low $T_{f}$ that yielded a low fraction of active TPBs.

FIB-SEM has been used to study degradation of Ni-YSZ anodes at elevated temperature. [9-12] Coarsening and sintering of the Ni phase in Ni-YSZ anodes has long been considered a key issue for solid oxide fuel cell (SOFC) durability. Accelerated anode ageing was carried out at higher than normal temperatures, $1000-1200{ }^{\circ} \mathrm{C}$. Measured particle size and TPB density changes with ageing time and temperature were fit with power-law coarsening. A combined coarsening-electrochemical model predicts changes in the anode polarization resistance reasonably well, and is extrapolated to make longterm predictions of Ni-YSZ anode structural and electrochemical performance evolution.

FIB-SEM has also been applied to study how the 3D structure of Li-ion battery positive electrodes impacts their charge-discharge characteristics. 3D reconstructions of $\mathrm{LiFePO}_{4}[13,14]$ and $\mathrm{LiCoO}_{2}[15$, 16] electrodes have been reported. In early work, only the oxide particles were resolved, because epoxy resin fillers used in sample preparation have poor contrast with carbonaceous electrode phases. Recently, silicone resin has been used as the filler, providing clear contrast between relevant phases. The 3D image data has been used to simulate lithiation-delithiation processes, providing realistic results by accounting for Li transport within oxide particles [17], within the electrolyte, and across interfaces. This allows one to compare the power and energy capabilities of different electrode structures; one example is the observed improvement in high $\mathrm{C}$-rate performance of $\mathrm{LiFePO}_{4}$ electrodes, where carbonate templating is used to improve the connectivity of the electronically conductive carbon network and 
introduce an array of micron-scale electrolyte spaces that help facilitate $\mathrm{Li}$ ion transport.

Several degradation effects are observed in $\mathrm{LiFePO}_{4} /$ carbon cathodes, using FIB-SEM to compare fresh and cycled cathodes, including cracks in the $\mathrm{LiFePO}_{4}$ particles, secondary disconnected particles, and agglomeration of carbon. [14] Low voltage (1 kV) SEM images showed that the carbon agglomerates have a different brightness than the fresh carbon, due to charging effects. This suggests that the electronic conductivity of the carbon agglomerates is low compared to that of the fresh carbon particles. HRTEM analysis shows that fresh carbon particles are quasi crystalline, whereas the LFP/carbon interface in the degraded electrode shows amorphous carbon surrounding the LFP particles. [18]

References:

[1] J.R. Wilson et al, Journal of Power Sources, 195 (2010) 1829-1840.

[2] J.R. Wilson et al, Electrochemistry Communications, 11 (2009) 1052-1056.

[3] J.S. Cronin et al, Journal of The Electrochemical Society, 159 (2012) B385.

[4] J.R. Wilson, J.S. Cronin, S.A. Barnett, Scripta Materialia, 65 (2011) 67-72.

[5] J.R. Wilson et al, Microsc Microanal, 15 (2009) 71-77.

[6] J.R. Wilson et al, Nat. Mater., 5 (2006) 541-544.

[7] J.R. Wilson, S.A. Barnett, Electrochemical and Solid-State Letters, 11 (2008) B181-B185.

[8] D. Kennouche et al, Physical Chemistry Chemical Physics, (2014).

[9] J.S. Cronin, J.R. Wilson, S.A. Barnett, Journal of Power Sources, 196 (2011) 2640-2643.

[10] D. Kennouche et al, Journal of Power Sources, 307 (2016) 604-612.

[11] D. Kennouche et al, Acta Materialia, 103 (2016) 204-210.

[12] D. Kennouche et al, Journal of the Electrochemical Society, 160 (2013) F1293-F1304.

[13] M. Ender et al, Journal of the Electrochemical Society, 159 (2012) A972-A980.

[14] R. Scipioni et al, Journal of Power Sources, 307 (2016) 259-269.

[15] Z. Liu et al, Journal of Power Sources, 227 (2013) 267-274.

[16] Z. Liu et al, Microsc Microanal, FirstView (2016) 1-9.

[17] A.H. Wiedemann et al, Electrochimica Acta, 88 (2013) 580 - 588.

[18] The authors gratefully acknowledge financial support from the National Science Foundation Ceramics Program, the Department of Energy Basic Energy Sciences Program, and the Office of Naval Research. 